\title{
A NEW CLASS OF ORTHOGONAL POLYNOMIALS: THE BESSEL POLYNOMIALS
}

\author{
BY \\ H. L. KRALL AND ORRIN FRINK
}

\section{INTRODUCTION}

The classical sets of orthogonal polynomials of Jacobi, Laguerre, and Hermite satisfy second order differential equations, and also have the property that their derivatives form orthogonal systems. There is a fourth class of polynomials with these two properties, and similar in other ways to the other three classes, which has hitherto been little studied. We call these the Bessel polynomials because of their close relationship with the Bessel functions of half-integral order. They are orthogonal, but not in quite the same sense as the other three systems. The Bessel polynomials satisfy:

$$
x^{2} \frac{d^{2} y}{d x^{2}}+(2 x+2) \frac{d y}{d x}=n(n+1) y .
$$

It will be shown that they occur naturally in the theory of traveling spherical waves.

These polynomials seem to have been considered first by S. Bochner [1](1), who pointed out their connection with Bessel functions. They are also mentioned in a paper by W. Hahn [2]. H. L. Krall [4] treated them as orthogonal polynomials in a generalized sense. In the present paper they are studied in much greater detail. We derive their recurrence relations, weight function, generating function, normalizing factors, and the analogue of the Rodrigues formula. We discuss also their relation to Bessel functions and to the spherical Bessel functions of Morse and Schelkunoff, as well as their applications to spherical waves.

This paper is in two parts. The first part deals with Bessel polynomials proper. The second part deals with generalized Bessel polynomials, which satisfy the differential equation:

$$
x^{2} y^{\prime \prime}+(a x+b) y^{\prime}=n(n+a-1) y .
$$

These specialize to the Bessel polynomials when $a=b=2$.

\section{Part I. Bessel polynomials}

1. The differential equation. We define the Bessel polynomial $y_{n}(x)$ to be the polynomial of degree $n$, and with constant term equal to unity, which

Presented to the Society, April 17, 1948; received by the editors December 3, 1947.

(1) Numbers in brackets refer to the references cited at the end of the paper. 
satisfies the differential equation:

$$
x^{2} \frac{d^{2} y}{d x^{2}}+(2 x+2) \frac{d y}{d x}=n(n+1) y,
$$

where $n=0,1,2, \cdots$. It is natural to extend this definition to negative subscripts by defining $y_{-n}(x)$ to be $y_{n-1}(x)$. Thus $y_{-1}(x)=y_{0}(x), y_{-2}(x)=y_{1}(x)$, and so on.

Similarly, we define the generalized Bessel polynomial $y_{n}(x, a, b)$ to be the polynomial of degree $n$, and with constant term equal to unity, which satisfies the differential equation:

$$
x^{2} \frac{d^{2} y}{d x^{2}}+(a x+b) \frac{d y}{d x}=n(n+a-1) y,
$$

where $n$ is a non-negative integer, provided $a$ is not a negative integer or zero, and $b$ is not zero. The special case $a=b=2$ gives the Bessel polynomials proper.

2. Explicit formulas for the Bessel polynomials. From the differential equation (1) we derive at once the formula:

$$
\begin{aligned}
y_{n}(x) & =\sum_{k=0}^{n} \frac{(n+k) !}{(n-k) ! k !}\left(\frac{x}{2}\right)^{k} \\
& =1+n(n+1) \frac{x}{2}+\cdots+\frac{(2 n-1) !}{(n-1) !}\left(\frac{x}{2}\right)^{n-1}+\frac{(2 n) !}{n !}\left(\frac{x}{2}\right)^{n} .
\end{aligned}
$$

For convenience of reference we list the first six of these polynomials:

$$
\begin{aligned}
& y_{0}(x)=1 \\
& y_{1}(x)=1+x \\
& y_{2}(x)=1+3 x+3 x^{2} \\
& y_{3}(x)=1+6 x+15 x^{2}+15 x^{3} \\
& y_{4}(x)=1+10 x+45 x^{2}+105 x^{3}+105 x^{4} \\
& y_{5}(x)=1+15 x+105 x^{2}+420 x^{3}+945 x^{4}+945 x^{5}
\end{aligned}
$$

These polynomials may be readily obtained from the recurrence relation:

$$
y_{n+1}=(2 n+1) x y_{n}+y_{n-1}
$$

which will be derived in $\$ 7$. Note that the coefficients of the Bessel polynomials are positive integers.

There is, of course, a second independent solution of the differential equation (1). This may be found by making the substitution $y=v \exp (2 / x)$, which gives the solution: $y=\exp (2 / x) y_{n}(-x)$; accordingly the general solution of (1) is: 


$$
y=A y_{n}(x)+B e^{2 / x} y_{n}(-x) .
$$

3. The wave equation in spherical coordinates. The wave equation

$$
\nabla^{2} u=\frac{1}{c^{2}} \frac{\partial^{2} u}{\partial t^{2}}
$$

in spherical coordinates $R, \theta, \phi, t$ becomes

$$
\frac{1}{R^{2}}\left[R \frac{\partial^{2}}{\partial R^{2}}(R u)+\frac{1}{\sin \theta} \frac{\partial}{\partial \theta}\left(\sin \theta \frac{\partial u}{\partial \theta}\right)+\frac{1}{\sin ^{2} \theta} \frac{\partial^{2} u}{\partial \phi^{2}}\right]=\frac{1}{c^{2}} \frac{\partial^{2} u}{\partial t^{2}} .
$$

If this is solved by separation of variables, the radial factor $f(R)$ is found to satisfy the differential equation:

$$
R^{2} \frac{d^{2} f}{d R^{2}}+2 R \frac{d f}{d R}+k^{2} R^{2} f=n(n+1) f
$$

where $k c=\omega$, and $\exp (i \omega t)$ is the time factor. (See J. A. Stratton. Electromagnetic theory, pp. 440-444.) If we let $r=k R$, this becomes:

$$
r^{2} \frac{d^{2} f}{d r^{2}}+2 r \frac{d f}{d r}+r^{2} f=n(n+1) f .
$$

It is well known that equation (6) may transformed into Bessel's equation by the substitution $f(r)=r^{-1 / 2} J(r)$, which yields:

$$
r^{2} J^{\prime \prime}+r J+r^{2} J=(n+1 / 2)^{2} J .
$$

If $n$ is an integer, the solutions of (7) are Bessel functions of half-integral order. It is well known that these are elementary functions.

However, it is simpler to treat equation (6) by making the substitution $f(r)=w(r) / r$, which gives the equation:

$$
r^{2}\left(w^{\prime \prime}+w\right)=n(n+1) w .
$$

Now real solutions of the wave equation found by separating the variables represent standing waves. Two or more such solutions must be combined to get traveling waves. There is, however, a standard method of obtaining traveling waves directly. This is to introduce the pure imaginary variable $z=i k R=i r$. Then real and imaginary parts of a single solution of the wave equation represent traveling waves. Accordingly, in (8) we let $z=i r$ $=i k R$ and $w(r)=e^{-z} y(z)=e^{-i r} y(i r)$. We then get:

$$
z^{2}\left(\frac{d^{2} y}{d z^{2}}-2 \frac{d y}{d z}\right)=n(n+1) y .
$$

For integral values of $n$, (9) has solutions which are polynomials in $1 / z$. Therefore we make the final substitution $x=1 / z=1 / i k R$, and obtain (1): 


$$
x^{2} y^{\prime \prime}+(2 x+2) y^{\prime}=n(n+1) y .
$$

This is the equation for Bessel polynomials. It follows that the wave equation in spherical coordinates has solutions of the form:

$$
u(R, \theta, \phi, t)=R^{-1} P_{n}^{m}(\cos \theta) \sin (m \phi-\alpha) e^{i(\omega L k R)} y_{n}(1 / i k R),
$$

where $y_{n}(x)=y_{n}(1 / i k R)$ is a Bessel polynomial, and $k c=\omega$. Note that the real and imaginary parts of (10) represent waves traveling in the radial direction with velocity $c$. Formula (10) makes evident the elementary nature of this type of solution, and suggests a treatment of spherical waves based on a theory of the functions $y_{n}(x)$ considered as a class of orthogonal polynomials, rather than on the usual theory of Bessel functions.

4. The relation of Bessel polynomials to Bessel functions. In the preceding $\$ 3$, equation ( 7 ) is the differential equation for Bessel functions of order $n+1 / 2$. Keeping track of the transformations connecting equations (1), (7), (8), and (9), and comparing coefficients, we find the following formulas connecting the Bessel polynomials and Bessel functions:

$$
\begin{aligned}
y_{n}(1 / i r) & =(\pi / 2)^{1 / 2} r^{1 / 2} e^{i r}\left[i^{-n-1} J_{n+1 / 2}(r)+i^{n} J_{-n-1 / 2}(r)\right] . \\
J_{n+1 / 2}(r) & =(2 \pi r)^{-1 / 2}\left[i^{-n-1} e^{i r} y_{n}(-1 / i r)+i^{n+1} e^{-i r} y_{n}(1 / i r)\right] . \\
J_{-n-1 / 2}(r) & =(2 \pi r)^{-1 / 2}\left[i^{n} e^{i r} y_{n}(-1 / i r)+i^{-n} e^{-i r} y_{n}(1 / i r)\right] .
\end{aligned}
$$

Schelkunoff (Electromagnetic waves, pp. 51-52) and P. M. Morse (Vibration and sound, pp. 246-247) define spherical Bessel functions $\bar{J}_{n}(r), \bar{N}\left(r_{n}\right)$, and $j_{n}(r), n_{n}(r)$, which are solutions of our differential equations (8) and (6) respectively. These functions are related to the Bessel functions of halfintegral order, and hence to the Bessel polynomials. The latter relations are:

$$
\begin{aligned}
y_{n}(-1 / i r) & =e^{-i r}\left[i^{n+1} \bar{J}_{n}(r)-i^{n} \bar{N}_{n}(r)\right], \\
\bar{J}_{n}(r) & =\left[i^{-n-1} e^{i r} y_{n}(-1 / i r)+i^{n+1} e^{-i r} y_{n}(1 / i r)\right] / 2, \\
\bar{N}_{n}(r) & =-\left[i^{-n} e^{i r} y_{n}(-1 / i r)+i^{n} e^{-i r} y_{n}(1 / i r)\right] / 2,
\end{aligned}
$$

in the case of the Schelkunoff functions $\bar{J}_{n}(r)$ and $\bar{N}_{n}(r)$. Likewise we have:

$$
\begin{aligned}
j_{n}(r) & =(1 / 2 r)\left[i^{-n-1} e^{i r} y_{n}(-1 / i r)+i^{n+1} e^{-i r} y_{n}(1 / i r)\right] . \\
n_{n}(r) & =(1 / 2 r)\left[i^{n} e^{i r} y_{n}(-1 / i r)+i^{-n} e^{-i r} y_{n}(1 / i r)\right] . \\
y_{n}(1 / i r) & =r e^{i r}\left[i^{-n-1} j_{n}(r)+i^{n} n_{n}(r)\right],
\end{aligned}
$$

for the Morse functions $j_{n}(r)$ and $n_{n}(r)$. These functions are connected with the Bessel functions of half-integral order by the relations:

$$
\begin{aligned}
& \bar{J}_{n}(r)=(\pi r / 2)^{1 / 2} J_{n+1 / 2}(r), \quad \bar{N}_{n}(r)=(-1)^{n+1}(\pi r / 2)^{1 / 2} J_{-n-1 / 2}(r) \\
& j_{n}(r)=(\pi / 2 r)^{1 / 2} J_{n+1 / 2}(r), \quad n_{n}(r)=(\pi / 2 r)^{1 / 2} J_{-n-1 / 2}(r) .
\end{aligned}
$$

5. Orthogonality and the weight function. We shall now show that the 
Bessel polynomials form an orthogonal system, the weight function being $e^{-2 / x}$ and the path of integration the unit circle in the complex plane. The weight function and the path are not unique; an arbitrary analytic function may be added to the weight function, the weight function may be multiplied by a nonzero constant, and the unit circle may be replaced by an arbitrary curve around the origin. Formula (10) shows that in applications to spherical waves we are interested in pure imaginary values of the argument $x$. This suggests a path of integration along the imaginary axis; but we find the unit circle a more convenient path for deriving formulas.

To prove orthogonality we first note that the differential equation (1) may be written in the form:

$$
\left(x^{2} e^{-2 / x} y_{n}^{\prime}\right)^{\prime}=n(n+1) e^{-2 / x} y_{n} .
$$

Integrating by parts counter-clockwise around the unit circle we find:

$$
n(n+1) \int_{U} y_{m} y_{n} e^{-2 / x} d x=\int_{U}\left(x^{2} e^{-2 / x} y_{n}^{\prime}\right)^{\prime} y_{m} d x=-\int_{U} x^{2} e^{-2 / x} y_{n}^{\prime} y_{m}^{\prime} d x
$$

Interchanging $m$ and $n$ and subtracting, we get:

$$
\int_{U} y_{m} y_{n} e^{-2 / x} d x=0, \quad m \neq n .
$$

This is the required orthogonality relation.

6. The analogue of Rodrigues' formula. Corresponding to Rodrigues' formula for the Legendre polynomials, and to similar well known formulas for the Hermite, Laguerre, and Jacobi polynomials, we now express the Bessel polynomials $y_{n}(x)$ in terms of the $n$th derivative of a multiple of the weight function $e^{-2 / x}$ :

$$
y_{n}(x)=2^{-n} e^{2 / x} \frac{d^{n}}{d x^{n}}\left(x^{2 n} e^{-2 / x}\right) .
$$

Here the factor $2^{-n}$ is to make the constant term equal to unity. Note that the right side of (18) is a polynomial of degree $n$. For $k<n$, this polynomial is orthogonal to $x^{k}$, with weight function and path as before, that is:

$$
\frac{1}{2 \pi i} \int_{U} x^{k} 2^{-n} \frac{d^{n}}{d x^{n}}\left(x^{2 n} e^{-2 / x}\right) d x=0,
$$

for $k=0,1,2, \cdots, n-1$. This may be seen by integrating by parts $k+1$ times around the unit circle. But by formula (17), the Bessel polynomial $y_{n}(x)$ is also orthogonal to $x^{k}$ with the same path and weight function. Since this property determines uniquely up to a multiplicative constant a polynomial of degree $n$, and since the constant terms are both unity, it follows that the two sides of (18) are identical. Formula (18) may also be verified directly, using the explicit formula (3). 
In the same manner it may be shown more generally that with $k$ an integer, $k^{(n)}=k(k-1) \cdots(k-n+1)$ :

$$
\frac{1}{2 \pi i} \int_{U} x^{k} y_{n}(x) e^{-2 / x} d x=\frac{(-2)^{k+1} k^{(n)}}{(n+k+1) !} .
$$

7. Recurrence relations for the Bessel polynomials. Among the infinitely many recurrence relations for the Bessel polynomials, we list the following five as being the most useful or interesting. They resemble in some ways the relations for the Bessel functions, and in other ways the relations for the classical orthogonal polynomials.

$$
\begin{aligned}
y_{n+1} & =(2 n+1) x y_{n}+y_{n-1}, \\
x^{2} y_{n}^{\prime} & =(n x-1) y_{n}+y_{n-1}, \\
x^{2} y_{n-1}^{\prime} & =y_{n}-(n x+1) y_{n-1}, \\
x\left(y_{n}^{\prime}+y_{n-1}^{\prime}\right) & =n\left(y_{n}-y_{n-1}\right), \\
(n x+1) y_{n}^{\prime}+y_{n-1}^{\prime} & =n^{2} y_{n} .
\end{aligned}
$$

The recurrence relations (21) may be derived from the well known recurrence relations for the Bessel functions, using the relations (11). They may also be derived from the explicit formula (3), or by making use of the orthogonality of the Bessel polynomials. The latter method is described below in $\$ 16$, where the recurrence relations (51) for the generalized Bessel polynomials are obtained. These correspond to the first two relations of (21), which are included as the special case where $a=b=2$. Consequently the details of the derivation of relations (21) will be omitted.

Other recurrence relations for the Bessel polynomials may be derived from the relations (21). It should be noted that these relations continue to hold for negative values of $n$, as may be verified by using the definition $y_{-n}(x)=y_{n-1}(x)$

8. The normalizing factor. If a function $f(x)$ is formally expanded in an infinite series of Bessel polynomials:

$$
f(x) \sim \sum_{n=0}^{\infty} f_{n} y_{n}(x),
$$

the coefficients $f_{n}$ are given by

$$
f_{n}=\frac{\int f(x) y_{n}(x) e^{-2 / x} d x}{\int y_{n}^{2}(x) e^{-2 / x} d x}
$$

Hence it is desirable to know the value of the integral in the denominator. We evaluate this integral by using formula (20) with $n=k$. This gives: 


$$
\frac{1}{2 \pi i} \int_{U} y_{n}^{2}(x) e^{-2 / x} d x=\frac{1}{2 \pi i} \int_{U} \frac{2 n !}{n !}\left(\frac{x}{2}\right)^{n} y_{n}(x) e^{-2 / x} d x=(-1)^{n+1} \frac{2}{2 n+1} .
$$

It is curious to note that except for the alternating sign, this is the same as the normalizing factor for the Legendre polynomials. It follows from (23) that the polynomials:

$$
\frac{(1+i) i^{n}}{2}\left(\frac{2 n+1}{2 \pi}\right)^{1 / 2} y_{n}(x)
$$

form an orthonormal set with weight function $e^{-2 / x}$ and path of integration the unit circle.

9. The generating function for Bessel polynomials. In view of formula (3), a generating function $G(x, t)$ of the usual kind would have the form:

$$
G(x, t)=\sum_{n=0}^{\infty} y_{n}(x) t^{n}=\sum_{n=0}^{\infty} \frac{(2 n) !(x t)^{n}}{n ! 2^{n}(1-t)^{2 n+1}} .
$$

However, since this series converges only for $t /(1-t)=0$, we replace $t^{n}$ by $t^{n} / n !$, which gives:

$$
\exp \left(\frac{1-(1-2 x t)^{1 / 2}}{x}\right)=\sum_{n=0}^{\infty} y_{n-1}(x) t^{n} / n ! .
$$

Hence the desired generating function is the function on the left side of (25). Formula (25) may be obtained from the following formulas due to Glaisher:

$$
\begin{aligned}
& \sum_{n=0}^{\infty} J_{n-1 / 2}(r) t^{n} / n !=(2 / \pi r)^{1 / 2} \cos \left(r^{2}-2 r t\right)^{1 / 2} \\
& \sum_{n=0}^{\infty} J_{1 / 2-n}(r) t^{n} / n !=(2 / \pi r)^{1 / 2} \sin \left(r^{2}+2 r t\right)^{1 / 2}
\end{aligned}
$$

These may be found in G. N. Watson, $A$ treatise on the theory of Bessel functions, p. 140. Multiplying the first relation of (11) by $t^{n} / n$ ! gives:

$$
\begin{aligned}
y_{n-1}(1 / i r) t^{n} / n ! & =(\pi r / 2)^{1 / 2} e^{i r}\left[i^{-n} J_{n-1 / 2}(r)+i^{n-1} J_{1 / 2-n}(r)\right] t^{n} / n ! \\
& =(\pi r / 2)^{1 / 2} e^{i r}\left[(t / i)^{n} J_{n-1 / 2}(r) / n !-i(i t)^{n} J_{1 / 2-n}(r) / n !\right] .
\end{aligned}
$$

Combining (26) and (27) then gives:

$$
\begin{aligned}
\sum_{n=0}^{\infty} y_{n-1}(1 / i r) t^{n} / n ! & =e^{i r}\left(\cos \left(r^{2}+2 i r t\right)^{1 / 2}-i \sin \left(r^{2}+2 i r t\right)^{1 / 2}\right) \\
& =e^{i\left(r-\left(r^{2}+2 i r t\right)^{1 / 2}\right.},
\end{aligned}
$$

from which formula (25) may be obtained. An interesting consequence of (25) is found by setting $t=z-x z^{2} / 2$ : 


$$
e^{z}=\sum_{n=0}^{\infty} y_{n-1}(x)\left(z-x z^{2} / 2\right)^{n} / n !
$$

Formula (25) for the generating function may also be verified directly, without the use of formulas from the theory of Bessel functions, by substituing the power series for $\left(1-(1-2 x t)^{1 / 2}\right) / x$ in the power series for $e^{z}$, and comparing coefficients with those given by formula (3).

10. The moments of the weight function. If we consider the weight function to be $e^{-2 / x} / 2 \pi i$ rather than $e^{-2 / x}$, then its moments, with the unit circle as path of integration, are given by:

$$
\alpha_{n}=\frac{1}{2 \pi i} \int_{U} x^{n} e^{-2 / x} d x=(-2)^{n+1} /(n+1) !
$$

In the theory of orthogonal polynomials it is often important to know the value of determinant:

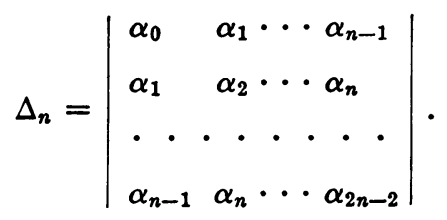

This determinant is discussed in H. L. Krall $[3,4]$. In particular we wish to verify that it is never zero, in order to conclude that polynomials which are orthogonal with the given weight function and path of integration are uniquely determined, up to a multiplicative constant, by this property. This was assumed in deriving the Rodrigues' formula in \$6. Accordingly, we substitute the value of $\alpha_{n}$ from (30) into (31) and evaluate the resulting determinant by repeated subtraction of rows. We omit the details of this evaluation and give only the result:

$$
\Delta_{n}=(-1)^{n(n-1) / 2}(-2)^{n^{2}} \prod_{s=1}^{n-1} s ! / \prod_{0=n}^{2 n-1} s !
$$

We conclude from formula (32) that $\Delta_{n}$ is never zero.

\section{Part II. Generalized Bessel polynomials}

11. The differential equation. As defined in $\$ 1$, the generalized Bessel polynomial $y_{n}(x, a, b)$ is the polynomial of degree $n$ and with constant term equal to unity, which satisfies the differential equation (2). Equation (1) for the Bessel polynomials proper is the special case of (2) where $a=b=2$. We assume that $b$ is not zero. With $b=0$, the general solution of (2) is $y=A x^{n}$ $+B x^{1-a-n}$. We exclude also the cases where $a$ is zero or a negative integer, since the theory developed below breaks down in those cases, and a special treatment is required. The value of the constant $b$ is not important, since re- 
placing $x$ by a constant multiple of $x$ changes $b$ without changing the rest of the equation.

Note that the derivative $y^{\prime}$ of a solution of (2) satisfies the equation:

$$
x^{2} \frac{d^{2} y^{\prime}}{d x^{2}}+(a x+2 x+b) \frac{d y^{\prime}}{d x}=n(n+a+1) y^{\prime},
$$

which has the form of (2), but with $a$ replaced by $a+2$. It follows that the derivatives of generalized Bessel polynomials are generalized Bessel polynomials, and also that the polynomials in the excluded cases where $a=0,-1$, $-2, \cdots$ can be obtained by repeated integration from the polynomials with $a=1$ or 2 .

We proceed now to develop a theory of the generalized Bessel polynomials roughly parallel to that given for the Bessel polynomials proper. When $a$ is an integer, the two theories are very similar. There are certain differences, however, when $a$ is nonintegral.

12. Explicit formulas for the generalized Bessel polynomials. Instead of the notation $y_{n}(x, a, b)$ for these polynomials, we shall of ten use the shorter forms $y_{n}(x)$ or $y_{n}$. Solving (2) in the usual manner we find the explicit formula:

$$
y_{n}(x, a, b)=\sum_{k=0}^{n} C_{n, k}(n+k+a-2)^{(k)}\left(\frac{x}{b}\right)^{k},
$$

where $C_{n, k}$ is a binomial coefficient, and $(x)^{(k)}$ as usual means $x(x-1) \cdots$ $(x-k+1)$. The first five of these polynomials are therefore given by:

$$
\begin{aligned}
y_{0}(x)= & 1 \\
y_{1}(x)= & 1+a\left(\frac{x}{b}\right) \\
y_{2}(x)= & 1+2(a+1)\left(\frac{x}{b}\right)+(a+1)(a+2)\left(\frac{x}{b}\right)^{2} \\
y_{3}(x)= & 1+3(a+2)\left(\frac{x}{b}\right)+3(a+2)(a+3)\left(\frac{x}{b}\right)^{2} \\
& +(a+2)(a+3)(a+4)\left(\frac{x}{b}\right)^{3}, \\
y_{4}(x)= & 1+4(a+3)\left(\frac{x}{b}\right)+6(a+3)(a+4)\left(\frac{x}{b}\right)^{2} \\
& +4(a+3)(a+4)(a+5)\left(\frac{x}{b}\right)^{3} \\
& +(a+3)(a+4)(a+5)(a+6)\left(\frac{x}{b}\right)^{4} .
\end{aligned}
$$


The coefficients are no longer necessarily integral, as was the case with the Bessel polynomials proper. Note that in the excluded cases $a=0,-1$, $-2, \cdots$ one or more of the polynomials $y_{n}(x)$ are of degree less than $n$.

If $a$ is not an integer, the second solution of (2) is not an elementary function, but it may be represented by an infinite series, or in terms of integrals of elementary functions.

13. The weight function and its moments. We shall show that the generalized Bessel polynomials form an orthogonal system, with path of integration an arbitrary curve surrounding the zero point, and with weight function $\rho(x)$ given by:

$$
\rho(x)=\frac{1}{2 \pi i} \sum_{n=0}^{\infty} \frac{\Gamma(a)}{\Gamma(a+n-1)}\left(-\frac{b}{x}\right)^{n} .
$$

The series in (36) clearly converges for all $x$ except zero. Expanding (36) gives:

$$
\begin{aligned}
\rho(x)= & \frac{1}{2 \pi i}\left[a-1+\left(-\frac{b}{x}\right)+\frac{1}{a}\left(-\frac{b}{x}\right)^{2}+\frac{1}{a(a+1)}\left(-\frac{b}{x}\right)^{3}\right. \\
& \left.+\frac{1}{a(a+1)(a+2)}\left(-\frac{b}{x}\right)^{4}+\cdots\right] .
\end{aligned}
$$

Formula (37) would clearly have to be modified for the excluded cases $a=0,-1,-2, \cdots$. Note that $\rho(x)$ reduces to the previous weight function $e^{-2 / x} / 2 \pi i$ for the case $a=b=2$ of Bessel polynomials proper.

The function $\rho(x)$ differs, except when $a=1$ or 2 , from the function $\sigma(x)$ given by

$$
\sigma(x)=x^{a-2} e^{-b / x} / 2 \pi i,
$$

which is the factor needed to make equation (2) self-adjoint, and which is therefore a natural candidate for a weight function. However, $\sigma(x)$ is multiple-valued when $a$ is not an integer, and this is inconvenient if we wish to integrate around the point $x=0$. The function $\sigma(x)$ satisfies the differential equation:

$$
\left(x^{2} \sigma\right)^{\prime}=(a x+b) \sigma,
$$

while $\rho(x)$ satisfies the related nonhomogeneous equation:

$$
\left(x^{2} \rho\right)^{\prime}=(a x+b) \rho-\frac{(a-1)(a-2)}{2 \pi i} x .
$$

These equations are the same if $a$ is 1 or 2 , which suggests that the special case where $a$ is 1 may be of equal importance with the case where $a$ is 2 which we have treated in Part $I$. 
Formula (36) for $\rho(x)$ may be obtained by finding the solution of equation (40) of the form $\rho(x)=\sum_{n=0}^{\infty} c_{n} x^{-n}$. However, formula (36) for $\rho(x)$ was actually arrived at as follows: The moments $\alpha_{n}=\int_{U} x^{n} \rho(x) d x$ for a set of orthogonal polynomials satisfying (2) obey the recurrence relation:

$$
(n+a-1) \alpha_{n}+b \alpha_{n-1}=0
$$

(see H. L. Krall $[3,4]$ ). Hence, taking $\alpha_{0}=-b$, the moments must be given by:

$$
\alpha_{n-1}=\frac{\Gamma(a)}{\Gamma(a+n-1)}(-b)^{n} .
$$

From (42) the coefficients in (36) may be obtained immediately by multiplying by $x^{n}$ and integrating around the unit circle. Conversely, it may be verified that the function $\rho(x)$ as given by (36) has the moments given by (42).

Since we now have a formula for the moments, we may calculate the determinant $\Delta_{n}$ as defined by formula (31) for the generalized Bessel polynomials. Substituting the value of $\alpha_{n}$ from (42) into (31) and evaluating as before by repeated subtraction of rows, we find:

$$
\Delta_{n}=(-1)^{n(n-1) / 2}(-b)^{n^{2}} \prod_{s=1}^{n-1} s ! / \prod_{s=n-2}^{2 n-3}(a+s)^{(s+1)} .
$$

It can be seen from (43) that $\Delta_{n}$ is never zero except in the excluded cases $a=0,-1,-2, \cdots$.

14. Orthogonality of the generalized Bessel polynomials. If equation (2) is multiplied by $\rho(x)$, it may be written in the form:

$$
\left(x^{2} \rho y_{n}^{\prime}\right)^{\prime}+x(a-1)(a-2) y_{n}^{\prime} / 2 \pi i=n(n+a-1) \rho y_{n} .
$$

This follows from the fact that $\rho(x)$ satisfies equation (40). If we now multiply equation (44) by $y_{k}$ and integrate around the unit circle we get:

$$
\begin{aligned}
n(n+a-1) \int_{U} y_{n} y_{k} \rho d x & =\int_{U}\left(x^{2} \rho y_{n}^{\prime}\right)^{\prime} y_{k} d x+\frac{(a-1)(a-2)}{2 \pi i} \int_{U} x y_{n}^{\prime} y_{k} d x \\
& =-\int_{U} x^{2} \rho y_{n}^{\prime} y_{k}^{\prime} d x .
\end{aligned}
$$

Interchanging $n$ and $k$ in (45) and subtracting gives:

$$
\int_{U} y_{n} y_{k} \rho d x=0, \quad n \neq k,
$$

which shows that the polynomials are orthogonal with $\rho(x)$ as weight function.

15. The Rodrigues' formula. For the generalized Bessel polynomials the analogue of Rodrigues' formula is: 


$$
y_{n}(x, a, b)=b^{-n} x^{2-a} e^{b / x} d^{n}\left(x^{2 n+a-2} e^{-b / x}\right) / d x^{n} .
$$

Since this involves the function $\sigma(x)$ defined by formula (38) rather than the weight function $\rho(x)$, it is not an easy consequence of orthogonality, as was the Rodrigues' formula (18) for Bessel polynomials proper. We shall prove it by expanding the right side of (47) and identifying it with formula (34) for $y_{n}(x, a, b)$. We get:

$$
\begin{aligned}
b^{-n} x^{2-a} e^{b / x} \frac{d^{n}}{d x^{n}}\left(x^{2 n+a-2} e^{-b / x}\right) \\
=b^{-n} x^{2-a} e^{b / x} \sum_{s=0}^{\infty} \frac{(-b)^{s}}{s !}(2 n+a-2-s)^{(n)} x^{n+a-2-s} \\
=\sum_{s=0}^{\infty} \sum_{t=0}^{\infty} \frac{(-1)^{s} b^{s+t}(2 n+a-2-s)^{(n)} x^{n-s-t}}{s ! t ! b^{n}} \\
=\sum_{k=0}^{\infty} \frac{b^{k-n} x^{n-k}}{k !} \sum_{s=0}^{k}(-1)^{s} C_{k, 8}(2 n+a-2-s)^{(n)}
\end{aligned}
$$

But since we have:

$$
\sum_{s=0}^{k}(-1)^{\bullet} C_{k, e}(A-s)^{(n)}= \begin{cases}0, & \text { for } k>n, \\ n^{(k)}(A-k)^{(n-k)}, & \text { for } k \leqq n,\end{cases}
$$

the final expression in (48) reduces to:

$$
\begin{aligned}
\sum_{k=0}^{n} \frac{x^{n-k}}{k ! b^{n-k}} n^{(k)}(2 n+a & -2-k)^{(n-k)} \\
& =\sum_{k=0}^{n} C_{n, k}(n+a-2-k)^{(k)}\left(\frac{x}{b}\right)^{k}=y_{n}(x, a, b) .
\end{aligned}
$$

Consequently we have (47).

16. Recurrence relations for the generalized Bessel polynomials. The recurrence relations given by formulas (21) have analogues for the generalized Bessel polynomials. However, some of these are much more complicated than those for the Bessel polynomials proper, although they simplify considerably for certain integral values of $a$. Because they are so complicated we shall give only the following two:

$$
\begin{aligned}
& (n+a-1)(2 n+a-2) y_{n+1} \\
& =\left[(2 n+a)(2 n+a-2)\left(\frac{x}{b}\right)+a-2\right](2 n+a-1) y_{n} \\
& \quad+n(2 n+a) y_{n-1}, \\
& \quad x^{2}(2 n+a-2) y_{n}^{\prime}=[n(2 n+a-2) x-b n] y_{n}+b n y_{n-1} .
\end{aligned}
$$


These correspond to the first two relations of (21), as may be seen by placing $a=b=2$. The first relation of (51) has the standard form:

$$
y_{n+1}=(A x+B) y_{n}+C y_{n-1},
$$

which is the usual type of recurrence relation for orthogonal polynomials. Since the method of determining the coefficients $A, B$, and $C$ by using orthogonality is familiar, we shall prove only the second relation of (51). The first relation may be proved in a similar manner.

To prove the second relation of (51), we first select the constant $A$ so that $x^{2} y_{n}^{\prime}-A x y_{n}$ is a polynomial of degree $n$. It then has an expansion of the form:

$$
x^{2} y_{n}^{\prime}-A x y_{n}=\sum_{k=0}^{n} B_{k} y_{k} .
$$

We now multiply both sides of (53) by $\rho y_{k}$ and integrate around the unit circle. Since $y_{n}^{\prime}$ is orthogonal, with weight function $x^{2} \rho$, to any polynomial of degree less than $n-1$, we find that all the coefficients $B_{k}$ are zero except $B_{n}$ and $B_{n-1}$. Hence:

$$
x^{2} y_{n}^{\prime}=\left(A x+B_{n}\right) y_{n}+B_{n-1} y_{n-1} .
$$

We now determine $A, B_{n}$, and $B_{n-1}$ by equating the coefficients of $x^{n+1}$ and $x^{n}$, as well as the constant terms, using formula (34). This gives:

$$
\begin{aligned}
n(2 n+ & a-2)^{(n)} x^{n+1} b^{-n}+n(n-1)(2 n+a-3)^{(n-1)} x^{n} b^{1-n}+\cdots \\
= & A(2 n+a-2)^{(n)} x^{n+1} b^{-n}+A n(2 n+a-3)^{(n-1)} x^{n} b^{1-n}+\cdots \\
& +B_{n}(2 n+a-2)^{(n)} x^{n} b^{-n}+\cdots+B_{n}+\cdots+B_{n-1} .
\end{aligned}
$$

Hence we have:

$$
\begin{aligned}
A & =\frac{n(2 n+a-2)^{(n)}}{(2 n+a-2)^{(n)}}=n \\
B_{n} & =\left(\frac{n(n-1)(2 n+a-3)^{(n-1)}}{(2 n+a-2)^{(n)}}-\frac{A n(2 n+a-3)^{(n-1)}}{(2 n+a-2)^{(n)}}\right) b \\
& =\frac{-b n}{2 n+a-2}, \\
B_{n-1} & =-B_{n}=\frac{b n}{2 n+a-2} .
\end{aligned}
$$

From (56) and (54) we get the second relation of (51). This completes the proof. Other recurrence relations may be derived and proved in the same manner.

17. The normalizing factor. As in $\S 8$, it is desirable to have formulas for 
$\int y_{n}^{2} \rho d x$ and $\int x^{k} y_{n} \rho d x$, corresponding to formulas (23) and (20). These are:

$$
\begin{aligned}
\int_{U} x^{k} y_{n}(x) \rho(x) d x & =\frac{(-b)^{k+1} k^{(n)}}{(k+a+n-1)^{k+n}}, \\
\int_{U} y_{n}^{2}(x) \rho(x) d x & =\frac{(-1)^{n+1}(b) n !}{(2 n+a-1)(n+a-2)^{(n-1)}} .
\end{aligned}
$$

We prove (57) as follows:

$$
\begin{aligned}
\int_{U} x^{k} y_{n}(x) \rho(x) d x & =\frac{1}{2 \pi i} \int_{U} \sum_{p=0}^{n} \sum_{s=0}^{\infty}(-1)^{s+1} C_{n, p} \frac{(n+p+a-2)^{(p)} x^{k+p-s-1}}{(s+a-1)^{(s)} b^{p-s-1}} \\
& =\sum_{p=0}^{n}(-1)^{k+p+1} C_{n, p} \frac{(n+p+a-2)^{(p)} b^{k+1}}{(k+p+a-1)^{(k+p)}} \\
& =\frac{(-b)^{k+1} k^{(n)}}{(k+a+n-1)^{(k+n)}}
\end{aligned}
$$

which gives the formula (57). Note that the right side of (57) is zero for $k<n$. We prove (58) from (57), using the orthogonality relation (46) together with the explicit formula (34) for $y_{n}(x)$. This gives:

$$
\int_{U} y_{n}^{2} \rho d x=(2 n+a-2)^{(n)} b^{-n} \int_{U} x^{n} y_{n} \rho d x,
$$

and replacing the integral on the right side by its value from (57) gives formula (58). It follows from (58) that the functions:

$$
\left[(2 n+a-1)(n+a-2)^{(n-1)} / b n !\right]^{1 / 2} i^{n+1} y_{n}(x)
$$

are orthonormal with weight function $\rho(x)$.

18. Conclusion. In Part I we have given a fairly complete theory of the Bessel polynomials proper. Other topics that suggest themselves in this case are the location of the roots of these polynomials, and the actual expansion of particular functions in terms of them.

It should be noted that formula (10), which expresses traveling spherical waves in terms of the Bessel polynomial $y_{n}(1 / i k R)$, indicates that this function, when multiplied by $e^{-i k R} / R$, bears the same relation to traveling spherical waves that the Hankel function, or Bessel function of the third kind, given by:

$$
H_{n}(k r)=J_{n}(k r)+i N_{n}(k r)
$$

bears to traveling cylindrical waves. Thus the Bessel polynomials may be thought of as spherical Hankel functions.

Our investigation of the generalized Bessel polynomials in Part II has not been quite so complete. In particular, we have not succeeded in finding a generating function for these polynomials. The general case is more difficult 
to treat, one reason being that here one does not have the entire theory of Bessel functions to rely on to derive results.

As we have indicated, the special case of the generalized Bessel polynomials where $a=1$ seems to have particular importance, and may deserve as detailed a treatment as we have given in Part $I$ for the case $a=2$. For example, the polynomials corresponding to integral values of $a$, whose theory is particularly simple, may all be obtained by differentiation from the polynomials with $a=1$ or 2 . We have as yet found no application for the case $a=1$, however.

The cases we have excluded where $a=0,-1,-2, \cdots$ may also be worth studying. More attention could be given to the theory of the second solution of the differential equation (2). The weight function $\rho(x)$, given by formula (36), may also be worth further investigation. It is definable in terms of the function $y(x, a)$ given by:

$$
y(x, a)=x^{a}\left(1 / a+x / a(a+1)+x^{2} / a(a+1)(a+2)+\cdots\right) .
$$

This function satisfies the differential equation: $y^{\prime}-y=x^{a-1}$, and is expressible for positive $a$ by the formula:

$$
y(x, a)=e^{x} \int_{0}^{x} z^{a-1} e^{-z} d z .
$$

It is thus related to the incomplete gamma function, and has various interesting properties.

It would also be useful to have weight functions with respect to which the Bessel functions are orthogonal with path of integration along the real axis or the imaginary axis. Since in the first recurrence relations of (21) and (51) the coefficients of $y_{n-1}$ are positive, it follows from known results that no non-negative weight function exists with path of integration along the real axis (see O. Perron, Die Lehre von den Kettenbrüchen, Leipzig 1929, p. 376). On the other hand it follows from results of Pólya [8] and Boas [14] that infinitely many weight functions of Stieltjes type $d o$ exist for the real axis.

Still another question concerns the solutions of the differential equations (1) and (2) for non-integral values of $n$. The solutions of (1) for half-integral $n$ are expressible in terms of the Bessel functions of integral order. For nonintegral values of $n$, the attempt to solve equations (1) and (2) in series of powers of $x$ leads to merely asymptotic series which are divergent. In the case of equation (1) with half-integral values of $n$, this procedure leads to the wellknown asymptotic series for the Bessel functions. The appearance of these asymptotic series is related to the fact that the equations (1) and (2) have a nonregular singular point at $x=0$. The differential equations for the classical orthogonal polynomials of Jacobi, Laguerre, and Hermite have no finite irregular singular points. 


\section{REFERENCES}

1. S. Bochner, Über Sturm-Liouvillesche Polynomsysteme, Math. Zeit. vol. 29 (1929) pp. 730-736.

2. W. Hahn, Über die Jacobischen Polynome und zwei verwandte Polynomklassen, Math. Zeit. vol. 39 (1935) pp. 634-638.

3. H. L. Krall, Certain differential equations for Tchebycheff polynomials, Duke Math. J. vol. 4 (1938) pp. 705-719.

4. - On derivatives of orthogonal polynomials. II, Bull. Amer. Math. Soc. vol. 47 (1941) pp. 261-264.

5. J. Meixner, Orthogonale Polynomsysteme mit einer besonderen Gestalt der erzeugenden Funktion, J. London Math. Soc. vol. 9 (1933) pp. 6-13.

6. P. M. Morse, Vibration and sound, New York, 1936.

7. O. Perron, Die Lehre von den Kettenbriichen, Leipzig, 1929.

8. G. P6lya, Sur l'indetermination d'un théorème voisin du problème des moments, C. R. Acad. Sci. Paris vol. 207 (1938) pp. 708-711.

9. S. A. Schelkunoff, Electromagnetic waves, New York, 1943.

10. J. A. Shohat and J. D. Tamarkin, The problem of moments, Mathematical Surveys, vol. 1, New York, 1943.

11. J. A. Stratton, Electromagnetic theory, New York, 1941.

12. G. Szegö, Orthogonal polynomials, Amer. Math. Soc. Colloquium Publications, vol. 23, 1939.

13. G. N. Watson, $A$ treatise on the theory of Bessel functions, Cambridge, 1944.

14. R. P. Boas, The Stieltjes moment problem for functions of bounded variation, Bull. Amer. Math. Soc. vol. 45 (1939) pp. 399-404.

The Pennsylvania State College, State College, Pa. 